\title{
Schedule-induced drinking is not ubiquitous: Effect of water-bottle location
}

\author{
J. D. KEEHN and EMOKE JOZSVAI \\ York University, Toronto, Canada
}

\begin{abstract}
After adaptation and shaping, barpresses of two groups of 4 food-deprived rats were reinforced at fixed intervals of $30 \mathrm{sec}$ (FI-30) for 27 sessions, each one 25 min long. Water bottles were available, $16 \mathrm{~cm}$ from the food source for one group and $29 \mathrm{~cm}$ from it for the other group. Three animals in the first group but only 1 in the second developed polydipsia. When the bottle positions were reversed for the groups for a further 10 sessions, high drinkers remained high drinkers but the low drinkers did not become polydipsic. These data show that schedule-induced drinking is not ubiquitous, and they also suggest that once an alternative adjunctive behavior is acquired, drinking does not easily displace it.
\end{abstract}

Schedule-induced polydipsia is excessive water intake by food- but not water-deprived organisms exposed to schedules of intermittent reinforcement. It is supernormal drinking (Falk, 1961; see Roper, 1981, for a review of controls for normality) that challenges the hegemony of physiological homeostasis, and it is also a problem for the two-process classification of behavior, because neither respondent nor operant procedures are explicit in its origination (Falk, 1972; but see Wetherington, 1982, for a review of conditioning processes involved in scheduleinduced drinking). The phenomenon is robust, stereotyped in magnitude and frequency (Keehn \& Stoyanov, 1986), and ubiquitous insofar as it occurs reliably in intervals between pellet deliveries (the interreinforcement interval) whenever water is available (Gilbert, 1974).

In Gilbert's (1974) study, access to water was permitted for only selected parts of the interpellet interval, so unavailability of water as well as unavailability of food might have contributed to water intake. In the present study, we tested the ubiquity of schedule-induced polydipsia by locating the drinking tube at different distances from the feeder without constraining drinking opportunities. So far as we know, food to water distance has never been examined as a determinant of schedule-induced drinking, although this distance has varied from a few centimeters (Falk, 1961) to over $60 \mathrm{~cm}$ (Reid \& Staddon, 1982; Staddon \& Ayres, 1975).

\section{METHOD}

\section{Subjects}

Eight male Sprague-Dawley white rats supplied by the Charles River Co., Quebec, were used. They were received in the laboratory at about 70 days of age and maintained in individual home cages with free access to food and water for $\mathbf{3 0}$ days, during which they were handled

Correspondence should be addressed to J. D. Keehn, Atkinson College, York University, 4700 Keele Street, Downsview, Ontario M3J 1P3, Canada. and weighed daily and divided into two groups of 4 . After that, they were reduced to $85 \%$ of their free feeding weights by limited rations over several days and maintained close to these weights by appropriate postsession feedings over the course of the experiment. At the start of training the weights of the respective subjects were $365 \mathrm{~g}(\mathrm{~N} 1), 357 \mathrm{~g}$ (N2), $340 \mathrm{~g}(\mathrm{~N} 3), 387 \mathrm{~g}$ (N4), $362 \mathrm{~g}$ (F1), $355 \mathrm{~g}$ (F2), $341 \mathrm{~g}$ (F3), and $341 \mathrm{~g}$ (F4).

\section{Apparatus}

Two standard two-bar Grason-Stadler rat boxes, $29 \times 23.5 \times 19 \mathrm{~cm}$ in size, were modified by the removal of one bar and fitted with graduated glass water bottles in one of two locations, one on the outside of the chamber door, beside the response bar, the other on the outside of the back wall, opposite the response bar. The metal spouts of the bottles were $5 \mathrm{~mm}$ in diameter and were angled into the chamber through a hole $5 \mathrm{~cm}$ above floor level such that the bottom of the spout was $2 \mathrm{~mm}$ inside the chamber. When in place, the bottle on the door (the near bottle) was $16 \mathrm{~cm}$ to the right of the food magazine; correspondingly, the bottle on the wall (the far bottle) was $29 \mathrm{~cm}$ across the chamber from the magazine. Only one bottle was in place at a time; when not in use, the second spout opening was covered with a metal plate mounted flush with the wall.

The response bar was situated above and to the left of a food magazine that presented 45-mg standard Noyes rat pellets when scheduled. Experimental sessions with each animal always occurred at the same time of day, and each animal was always trained in the same experimental chamber with the same water spout. Spout contacts were routed via Grason-Stadler drinkometers to a Microcomputer Control System (Micro Interfaces) that also controlled the scheduling of reinforcers and sessional data printouts. The chambers were mounted in chests with ventilating fans that masked extraneous noises.

\section{Procedure}

After preliminary adaptation and barpress training with successive approximations, the subjects were maintained for 2 baseline sessions under a continuous reinforcement schedule in which every barpress was reinforced. Sessions terminated when an animal had secured 50 reinforcers. Following this, there were 37 sessions in which reinforcers were scheduled at fixed intervals of $30 \mathrm{sec}$ (FI-30 sec). For the first 27 experimental sessions, the water bottle was in the near position for Subjects N1 through N4 (Group N), and in the far position for Subjects F1 through F4 (Group F). For Sessions 28 through 37, the water-bottle positions were reversed: far for Group $\mathbf{N}$ and near for Group $\mathbf{F}$.

Experimental sessions were conducted on 5 days each week, and each session lasted until an animal secured 50 reinforcers. Session water intakes were measured in situ from graduations on the water bottles, and latencies from reinforcement to the first lick were recorded and averaged at the end of a session by computer. 

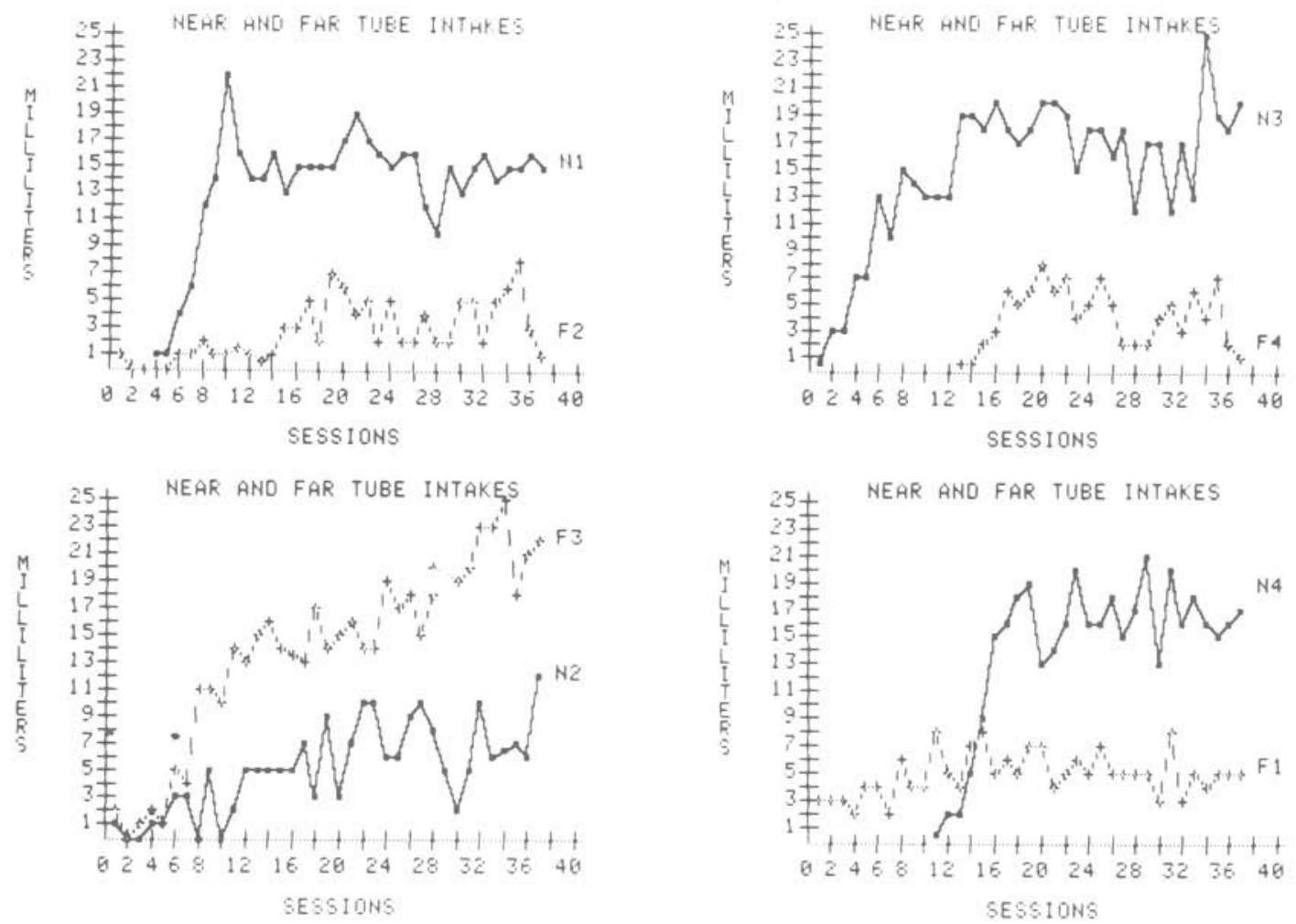

Figure 1. Water consumptions of the individual animals at the near and far tubes. Water-bottle positions were switched after Session 27.
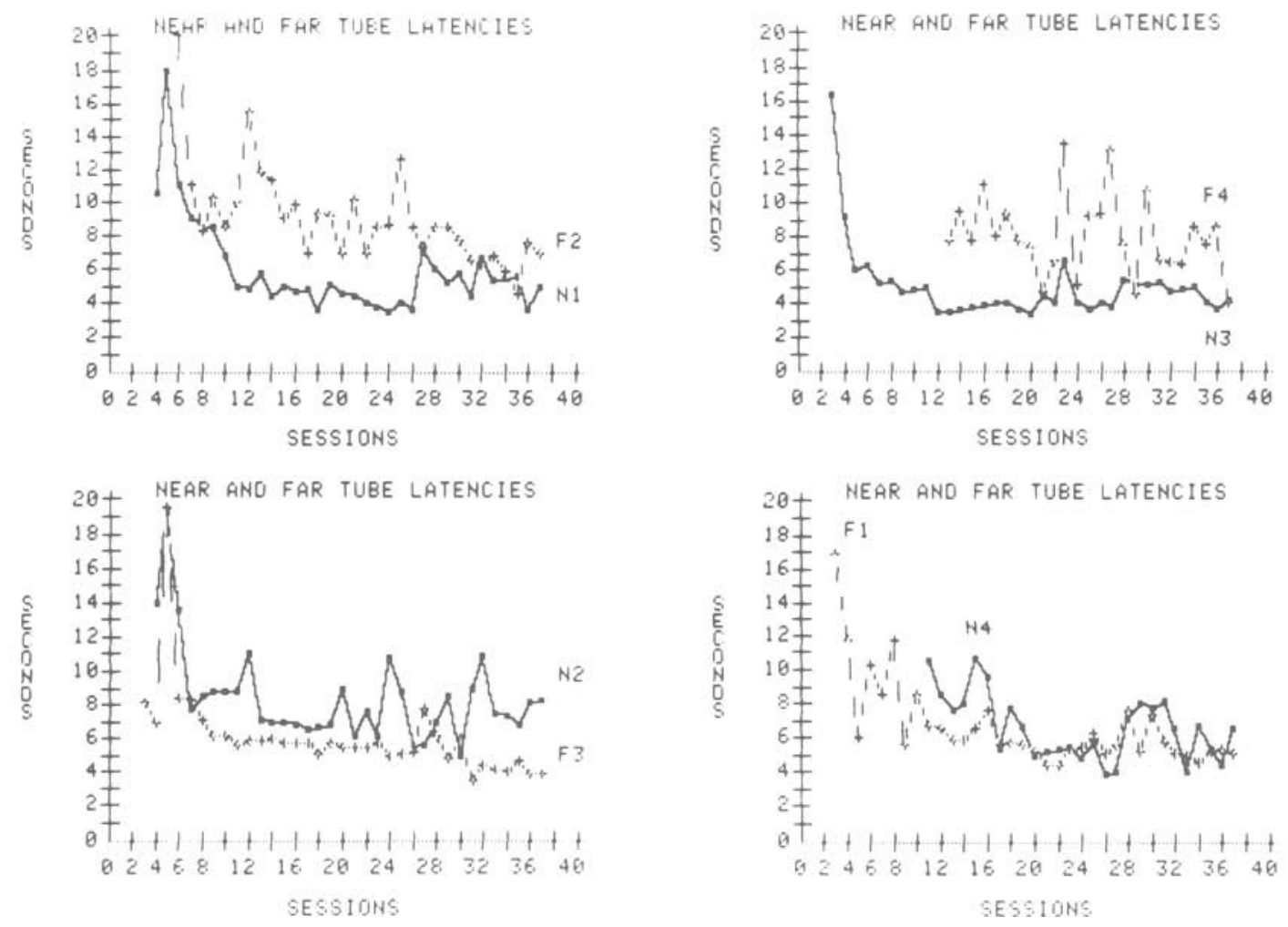

Figure 2. Latencies from reinforcement to first lick at the near and far tubes when drinking occurred. Water-bottle positions were switched after Session 27. 


\section{RESULTS}

Water intakes in baseline sessions were zero, except for $.5 \mathrm{ml}$ by $\mathrm{N} 1$ and $2 \mathrm{ml}$ by $\mathrm{N} 4$ on the first session. Subsequent water intakes by the individual animals (plotted in pairs by weight, one from each group) are shown in Figure 1 for each experimental session. In these sessions, water was available at the near location for Group $\mathrm{N}$ and at the far location for Group F for Sessions 1-27, and in the reverse positions for Sessions 28-37. Figure 1 shows, over Sessions 1-27, rapid acquisition of schedule-induced drinking by $3(\mathrm{~N} 1, \mathrm{~N} 3, \mathrm{~N} 4)$ of the 4 animals in Group N, but by only 1 (F3) of the 4 animals in Group F. The tubeposition reversal for the respective groups in Sessions 28-37 had no significant effect-the high drinkers remained high drinkers and the low drinkers remained low drinkers. Overall, in the last 3 sessions under each experimental condition, Group $\mathrm{N}$ averaged $14.2 \mathrm{ml}$ of water intake from the near bottle and $14.3 \mathrm{ml}$ from the far bottle; corresponding intakes by Group F were 8.2 and $7.4 \mathrm{ml}$, respectively. Thus, although bottle position affected the acquisition of schedule-induced drinking, neither group as a whole showed any effect of a change in water-bottle location as measured by amount of water consumed, although there were some individual differences.

Figure 2 shows mean latencies of the first lick whenever drinking occurred after reinforcement. In all cases, excepting animal F4, eat-drink latencies decreased with training over Sessions 1-27 regardless of the drink-tube location, and the most prolific drinkers drank with the shortest latencies. Animal F1, however, drank quickly after reinforcement, although it consumed relatively little water.

\section{DISCUSSION}

The proximity of the drinking tube to the food magazine makes a clear difference for the speed of development of schedule-induced water intake. Three of 4 animals trained initially with the near bottle reached high levels of water intake; only one of those trained first at the far bottle did. However, a change in the position of the bottle had minimal effect on water intake, either upwards or downwards, or in the time from reinforcement to the first lick. Schedule-induced polydipsia is not ubiquitous; even a small separation of food and water sources prevents or retards its acquisition.

Sometimes schedule-induced polydipsia is acquired rapidly and appears to be unlearned (Falk, 1961, 1972), and sometimes it accrues gradually, in the manner of a learned response (Keehn \& Stoyanov, 1986). Normally, food and water sources are only a few centimeters apart (Falk, 1961), and then schedule-induced polydipsia appears to be ubiquitous; but the ubiquity depends upon the animal's finding water. Obviously, without this contingency, schedule-induced drinking cannot occur. If food and water are widely separated, and many behaviors other than drinking are possible, then polydipsic drinking should be slow to develop and individual differences should occur. With food and water sources over $60 \mathrm{~cm}$ apart, this is what Staddon and Ayres (1975) found. In the present study, opportunities for other behaviors to compete with drinking were minimal, but they were sufficient to retard the acquisition of schedule-induced polydipsia by several animals.

\section{REFERENCES}

FALK, J. L. (1961). Production of polydipsia in normal rats by an intermittent food schedule. Science, 133, 195-196.

FALK, J. L. (1972). The nature and determinants of adjunctive behavior. In R. M. Gilbert \& J. D. Keehn (Eds.), Schedule effects: Drugs, drinking and aggression (pp. 148-173). Toronto: University of Toronto Press.

GILBERT, R. M. (1974). Ubiquity of schedule-induced polydipsia. Journal of the Experimental Analysis of Behavior, 21, 277-284.

Keehn, J. D., \& Stoyanov, E. (1986). The development of adjunctive drinking by rats: Conditioned and unconditioned components. Animal Learning \& Behavior, 14, 411-415.

REID, A. K., \& STADDON, J. E. R. (1982). Schedule-induced drinking: Elicitation, anticipation, or behavioral interaction? Journal of the Experimental Analysis of Behavior, 38, 1-18.

ROPER, T. J. (1981). What is meant by the term "schedule-induced," and how general is schedule induction? Animal Learning \& Behavior, 9, 433-440.

Staddon, J. E. R., \& Ayres, S. L. (1975). Sequential and temporal properties of behavior induced by a schedule of periodic food delivery. Behaviour, 54, 26-49.

WETHERINGTON, C. L. (1982). Is adjunctive behavior a third class of behavior? Neuroscience \& Biobehavioral Reviews, 6, 329-350. 